\title{
ГИПОГРАВИТАЦИЯ КАК ФАКТОР РИСКА ПОВЫШЕНИЯ УРОВНЯ ВНУТРИГЛАЗНОГО ДАВЛЕНИЯ
}

\author{
М. А. Валях ${ }^{\square}$, Д. В. Кац', Н. Г. Глазко², М. В. Баранов
}

1 Российский национальный исследовательский медицинский университет имени Н. И. Пирогова, Москва, Россия

2 Городская клиническая больница № 15 имени О. М. Филатова, Москва, Россия

${ }^{3}$ Научно-исследовательский институт космической медицины, Москва, Россия

\begin{abstract}
Космическая медицина давно занимается исследованием воздействия условий измененной гравитации на организм человека. За последнее время все больше внимания исследователи уделяют изменениям со стороны органа зрения. В первую очередь, это связано с увеличением жалоб космонавтов на недостаточную остроту зрения во время и после окончания космических полетов. Среди наиболее важных изменений у них было обнаружено повышение внутриглазного давления (ВГД) - наиболее опасная патология, нередко приводящая к необратимой слепоте за счет поражения зрительного нерва. Целью исследования было выяснить влияние измененной гравитации на офтальмотонус. Для этого 48 здоровых мужчин, средний возраст которых не превышал 22 года, были разделены на две равные по численности группы: группу контроля и группу, в которой моделировали условия гипогравитации путем помещения испытуемых в ортостатическое положение на время всего эксперимента (21 сутки). Измерение ВГД проводили в четырех контрольных точках эксперимента с помощью тонометра Маклакова. Была использована Z-аппроксимация Т-критерия Уилкоксона. Среднее

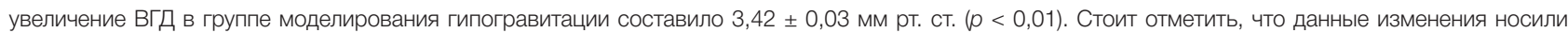
транзиторный характер и после окончания воздействия условий измененной гравитации показатели вернулись К исходным значениям.
\end{abstract}

Ключевые слова: ортостатическое пложение тела, гипогравитация, космический полет, внутриглазное давление

Благодарности: доценту кафедры офтальмологии имени академика А. П. Нестерова лечебного факультета, кандидату медицинских наук Кацу Д. В. из РНИМУ имени Н. И. Пирогова за редакцию статьи; заместителю директора, кандидату медицинских наук Баранову М. В. из НИИ космической медицинь за помощь в подборе испытуемых; врачу-офтальмологу Глазко Н. Г. из ГКБ № 15 имени О. М. Филатова за обработку полученных данных.

Информация о вкладе авторов: М. А. Валях - анализ литературы, сбор, анализ и интерпретация данных, подготовка рукописи; Н. Г. Глазко обработка полученных данных; М. В. Баранов - подбор испытуемых; Д. В. Кац - редактирование текста статьи.

Соблюдение этических стандартов: исследование одобрено этическим комитетом РНИМУ имени Н. И. Пирогова (протокол № 150 от 14 декабря 2015 г.).

$\bowtie$ Для корреспонденции: Максим Андреевич Валях

ул. Вешняковская, д. 23, г. Москва, 111539; maxvalyakh@gmail.com

Статья получена: 03.06.2019 Статья принята к печати: 17.06.2019 Опубликована онлайн: 19.06.2019

DOI: $10.24075 /$ vrgmu.2019.041

\section{HYPOGRAVITY AS A RISK FACTOR FOR INCREASED INTRAOCULAR PRESSURE}

Valyakh MA ${ }^{1 凶}$, Kats $D V^{1}$, Glazko NG², Baranov MV³

${ }_{1}^{1}$ Pirogov Russian National Research Medical University, Moscow, Russia

2 Filatov City Clinical Hospital № 15, Moscow, Russia

${ }^{3}$ Research Institute for Space Medicine, Moscow, Russia

Space medicine has long studied the impact of reduced gravity on the human body. Increasing complaints of insufficient visual acuity during and after space flights have been recently drawing a lot of attention to the effects of hypogravity on astronauts' vision. Abnormally high intraocular pressure (IOP) is one of the most clinically important changes occurring during space missions. It is a serious condition that often causes irreversible damage to the optic nerve and blindness. The aim of this study was to explore the effect of reduced gravity on IOP. The study recruited 48 young healthy men with the mean age of 22 years, who formed 2 equally sized groups. In the experimental group, hypogravity was simulated by placing the subjects into the orthostatic position for 21 days. IOP was measured at 4 time points using a Maklakov tonometer. Z-approximation of the Wilcoxon T test was applied. The average increase in IOP in the experimental group was $3.42 \pm 0.03 \mathrm{mmHg}$ $(p<0.01)$. The changes were, however, transient, and IOP levels went back to normal right after the exposure to hypogravity conditions was terminated.

Keywords: orthostatic body position, hypogravity, space flight, intraocular pressure

Acknowledgement: the authors thank Kats DV, Cand. Sci (Med), Assistant Professor at the Department of Ophthalmology of Pirogov Russian National Research Medical University, for revising the manuscript; Baranov MV Cand. Sci (Med), Vice Principal of Research Institute for Space Medicine, for helping with the recruitment process; Glazko NG, ophthalmologist at Filatov City Clinical Hospital No. 15, for his assistance with data analysis.

Author contribution: Valyakh MA — literature analysis, data acquisition, analysis and interpretation, manuscript preparation; Glazko NG — data analysis; Baranov MV — recruitment of participants; Kats DV — manuscript revision.

Compliance with ethical standards: the study was approved by the Ethics Committee of Pirogov Russian National Research Medical University (Protocol № 150 dated December 14, 2015)

$\bowtie$ Correspondence should be addressed: Maxim A. Valyakh

Veshniakovskaya 23, Moscow, 111539; maxvalyakh@gmail.com

Received: 03.06.2019 Accepted: 17.06.2019 Published online: 19.06.2019

DOI: $10.24075 /$ brsmu.2019.041

Влияние измененной гравитации на организм человека во время космических экспедиций изучают достаточно давно. Однако внимание изменениям со стороны органа зрения у космонавтов стали уделять только в последнее время. В частности, были зафиксированы подъемы внутриглазного давления (ВГД) в условиях измененной гравитации, а именно в условиях сниженной гравитационной силы гипогравитации. Данный вид гравитации обнаружен на Луне и предположительно на других планетах нашей Солнечной системы [1-5].

Первые данные о повышении ВГД во время космических полетов были получены с помощью ручного 
аппланационного тонометра: было зафиксировано увеличение ВГД на 20-25\% в течение первого часа от начала экспедиции [6].

Рядом других исследователей с помощью аппланационного тонометра Tono-pen был выявлен подъем уровня ВГД больше, чем у половины астронавтов во время орбитального полета [7].

Учитывая данные зарубежных коллег об изменениях со стороны органа зрения во время космических полетов, а также возросшую частоту космических экспедиций в последнее время и то, что увеличение ВГД может приводить к необратимому повреждению нервных волокон диска зрительного нерва (ДЗН), вызывая тем самым снижение зрения и/или слепоту, было принято решение о проведении клинического эксперимента с целью выявления взаимосвязи между гипогравитацией и уровнем офтальмотонуса. Стоит отметить, что помимо повышения уровня ВГД были зафиксированы и другие изменения со стороны органа зрения в процессе проведения исследований иностранными учеными, однако ни одно из них не было столь значимо, как увеличение уровня офтальмотонуса.

\section{ПАЦИЕНТЫ И МЕТОДЫ}

Данное исследование проводили в июле 2016 г. на базе кафедры офттальмологии лечебного факультета им. академика А. П. Нестерова РНИМУ им. Н. И. Пирогова, в ГКБ № 15 им. О. М. Филатова при участии ФГБУ ФНКЦ ФМБА России в НИИ космической медицины.

Для участия в эксперименте были отобраны 48 участников (96 глаз) молодого возраста. Критерии включения в исследование: 1) мужчины в возрасте 18-35 лет; 2) хорошая физическая подготовка; 3) нормальная рефракция глаза эметропия или миопия слабой или средней степени (до -6 диоптрий). Критерии исключения: 1) наличие острых офтальмологических заболеваний; 2) наличие дистрофии роговицы; 3) миопия высокой степени (от -6 дипотрий и выше); 4) наличие в анамнезе операций на роговице; 5) наличие иных соматических паталогий; 6) иной возраст и пол. Все участники были разделены на две равные группы путем открытой сравнительной рандомизации (метод конвертов).

Статистическую обработку данных проводили с помощью программы Statistica 8.0 (StatSoft Inc.; США). Приводимые параметры, имеющие нормальное распределение, представлены в формате: $M \pm m$, где М - среднее значение, $m$ - ошибка среднего значения. Для попарного сравнения двух независимых выборок применяли Z-аппроксимацию U-критерия МаннаУитни, для повторных внутригрупповых сравнений Z-аппроксимацию Т-критерия Уилкоксона. Критический уровень значимости при проверке статистических гипотез принимали равным < 0,05.

В 1-й группе были созданы условия, имитирующие нахождение в гипогравитации. Таких условий достигали за счет помещения испытуемых в ортостатическое положение, т. е. с углом наклона головного конца тела $+9,6^{\circ}$ относительно горизонтальной оси на дневной период и горизонтального положения в ночной период. Такое положение испытуемых в пространстве сохраняли в течение 21 суток. Обследуемые находились в помещениях, где была создана полная изоляция от внешних раздражающих факторов: повышена звукоизоляция, плотно закрыты окна. В помещении с испытуемыми могли находиться только представители медицинского персонала, участвующие в эксперименте; родственников и/или друзей не допускали. В строго регламентированное время было разрешено пользоваться телефоном и компьютером, читать. Обследования, процедуры личной гигиены, а также прием пищи в течение 21 дня наблюдения можно было проводить тоже в горизонтальном положении, не вставая с кровати.

Во 2-й группе, которая получила название «контроль», отсутствовали ограничения положения в пространстве, в ночной период (с 23:00 до 8:00) обследуемые находились в горизонтальном положении. Наблюдение длилось в течение 21 суток, регламент проведения обследований совпадал с регламентом в другой группе. Испытуемые имели право свободно перемещаться по территории, где проходил эксперимент, видеться с родственниками и друзьями, не находились в условиях повышенной изоляции от внешних раздражающих факторов (звукоизоляция была стандартная, окна не закрыты) (табл. 1).

Во время исследования были определены четыре основные точки: 1) первая точка, носившая название исходного измерения и/или фона - перед началом эксперимента (за день до помещения испытуемых в соответствующие условия); 2) вторая точка - на 11-е сутки эксперимента; 3) третья - на 21-е сутки; 4) четвертая точка - на первые сутки после окончания эксперимента (следующий день после прекращения нахождения в соответствующих условиях).

Для выявления изменений со стороны гидродинамики глаза всем испытуемым проводили измерение ВГД в установленные временные точки в утренние часы, когда повышение уровня ВГД достигает максимальных значений.

Помимо данного исследования всем пациентам проводили прямую офтальмоскопию в установленные контрольные точки, а также компьютерную периметрию до начала эксперимента и по его окончании с целью выявления возможных повреждений волокон зрительного нерва.

\section{Измерение уровня ВГД}

Исследование проводили с помощью аппланационного тонометра Маклакова (ПАО «Красногвардеец»; Россия), используя груз 10 г. Полученные данные были оценены с помощью переводной линейки Нестерова-Егорова и

Таблица 1. Общая характеристика групп исследования

\begin{tabular}{|c|c|c|c|}
\hline \multirow{2}{*}{ Группы } & \multicolumn{3}{|c|}{ Признак } \\
\cline { 2 - 4 } & $\begin{array}{c}\text { Количество } \\
\text { пациентов/глаз }\end{array}$ & Положение испытуемых в пространстве & $\begin{array}{c}\text { Средний возраст средней ошибкой (лет) } \\
\text { 1-я группа: } \\
\text { модель гипогравитации }\end{array}$ \\
\hline 2-я группа: контроль & $24 / 48$ & $\begin{array}{c}\text { Чередование ортостатического положения с углом наклона } \\
\text { тела }+9,6{ }^{\circ} \text { в дневной период и горизонтального положения } \\
\text { на ночной период (1-21 сутки наблюдения) }\end{array}$ & $21,75 \pm 3,83$ \\
\hline
\end{tabular}


представлены в значениях, равных показателям истинного (определяет действительный уровень офтальмотонуса и общепринятого в мировой офттальмологической практике) ВГД (Р, мм рт. ст.). Нормальный уровень истинного ВГД $\left(\mathrm{P}_{0}\right)$ при измерении ВГД тонометром Маклакова весом 10 г достигает 10-22 мм рт. ст.

\section{Прямая офтальмоскопия}

Исследование глазного дна проводили при помощи электронного офтальмоскопа ВХа, регистрационный номер ФС № 2005/1022 (NEITZ; Япония). Измерения проводили на узких зрачках без использования капель, вызывающих медикаментозный мидриаз во время эксперимента, и в состоянии медикаментозного мидриаза до начала эксперимента и на первые сутки после его окончания.

\section{Компьютерная периметрия}

Оценку полей зрения проводили методом статической периметрии на приборе Humphrey Field Analyzer II 750i (Zeiss; Германия), регистрационный номер ФС3 № 2008/02964. При анализе полученных данных учитывали показатели достоверности проведенного исследования: число ложноположительных и ложноотрицательных ответов, данные о потере фиксации взора.

\section{РЕЗУЛЬТАТЫ ИССЛЕДОВАНИЯ}

При анализе данных, полученных в ходе эксперимента, в 1-й группе (группа «модель гипогравитации») отмечается статистически значимое повышение уровня офтальмотонуса у всех испытуемых, которое было зафиксировано во время измерения ВГД на 11-е сутки, равное 3,33 \pm 0,08 мм рт. ст. На 21-е сутки эксперимента было отмечено продолжение увеличения уровня ВГД (3,42 \pm 0,03 мм рт. ст.) по сравнению с данными, полученными до начала эксперимента. Однако стоит отметить, что на первые сутки после окончания эксперимента значения ВГД вернулись к значениям, соотносимым со значениями, полученными до начала эксперимента (табл. 2).

При оценке результатов прямой офтальмоскопии на протяжении всего эксперимента ни у одного испытуемого не было зафиксировано отклонений от нормы. Диск зрительного нерва (ДЗН) - бледно-розовый, экскавация физиологическая $(0,3-0,4)$, сосудистый пучок в центре, ход и калибр сосудов не изменен, макулярная область без особенностей, на периферии зон дистрофии и/или разрывов нет.

По данным компьютерной периметрии, грубых нарушений в виде абсолютных скотом или значительного увеличения слепого пятна при анализе показателей испытуемых в этой группе выявлено не было.

В группе «контроль» никаких изменений уровня ВГД во время эксперимента и по его окончании не было зафиксировано. Значения у всех испытуемых были в пределах нормы на протяжении всего исследования (табл. 3). Так же как и в первой группе при оценке данных прямой офтальмоскопии и компьютерной периметрии, отклонений от нормы зафиксировано не было.

Статистический анализ изменений ВГД, зафиксированных в исследуемых группах с помощью U-критерия МаннаУитни для двух несвязанных совокупностей, позволил определить следующее: для групп «модель гипогравитации» и «контроль» $U_{\text {эмп. }}=0$, тогда как $U_{\text {крт. }}=834(p<0,01)$, что говорит о статистической достоверности и значимости полученных результатов.

Таблица 2. Исследование ВГД в группе «модель гипогравитации»

\begin{tabular}{|c|c|c|}
\hline \multirow{2}{*}{ Срок исследования } & \multicolumn{2}{|l|}{ Параметр } \\
\hline & \multicolumn{2}{|c|}{ Среднее ВГД со средней ошибкой, мм рт. ст. } \\
\hline Исходное & \multicolumn{2}{|l|}{$15,75 \pm 0,72$} \\
\hline \multirow[b]{2}{*}{ 11-е сутки } & \multicolumn{2}{|l|}{$19,08 \pm 0,64$} \\
\hline & $\Delta$ исходное - 11-е сутки эксперимента & $\begin{array}{c}3,33 \pm 0,08 \\
p<0,01\end{array}$ \\
\hline \multirow[b]{2}{*}{ 21-е сутки } & \multicolumn{2}{|l|}{$19,17 \pm 0,69$} \\
\hline & $\begin{array}{c}\Delta \text { исходное - 21-е сутки } \\
\text { эксперимента }\end{array}$ & $\begin{array}{c}3,42 \pm 0,03 \\
p<0,01\end{array}$ \\
\hline \multirow[b]{2}{*}{ Первые сутки после выхода испытуемых из эксперимента } & \multicolumn{2}{|l|}{$15,67 \pm 0,62$} \\
\hline & $\Delta$ исходное - 1-е сутки после окончания эксперимента & $\begin{array}{l}0,08 \pm 0,1 \\
p>0,05\end{array}$ \\
\hline
\end{tabular}

Таблица 3. Исследование ВГД в группе «контроль»

\begin{tabular}{|c|c|c|}
\hline \multirow{2}{*}{ Срок исследования } & \multicolumn{2}{|l|}{ Параметр } \\
\hline & \multicolumn{2}{|c|}{ Среднее ВГД со средней ошибкой, мм рт. ст. } \\
\hline Исходное & \multicolumn{2}{|l|}{$15,75 \pm 0,72$} \\
\hline \multirow[b]{2}{*}{ 11-е сутки } & \multicolumn{2}{|l|}{$15,79 \pm 0,73$} \\
\hline & $\Delta$ исходное - 11-е сутки эксперимента & $\begin{array}{c}0,04 \pm 0,01 \\
p>0,05\end{array}$ \\
\hline \multirow[b]{2}{*}{ 21-е сутки } & \multicolumn{2}{|l|}{$15,71 \pm 0,71$} \\
\hline & $\Delta$ исходное - 21-е сутки эксперимента & $\begin{array}{c}0,04 \pm 0,01 \\
p>0,05\end{array}$ \\
\hline \multirow[b]{2}{*}{ Первые сутки после выхода испытуемых из эксперимента } & \multicolumn{2}{|l|}{$15,77 \pm 0,71$} \\
\hline & $\Delta$ исходное - 1-е сутки после окончания эксперимента & $\begin{array}{c}0,02 \pm 0,01 \\
p>0,05\end{array}$ \\
\hline
\end{tabular}




\section{ОБСУЖДЕНИЕ РЕЗУЛЬТАТОВ}

Гипогравитация оказывает влияние на офтальмотонус, приводя к повышению ВГД. Во время клинического эксперимента были получены статистически достоверные и значимые результаты повышения ВГД. Среднее увеличение данного показателя составило 3,42 \pm 0,03 мм рт. ст. Однако можно предположить, что влияние гипогравитации на офтальмотонус носит транзиторный характер, так как уже в первые сутки после окончания эксперимента происходит восстановление уровня ВГД.

Данные изменения можно объяснить тем, что во время нахождения в условиях моделирования гипогравитации в организме испытуемых происходит перераспределение жидкости, сопровождающееся увеличением кровенаполнения структур и органов головы и шеи, в том числе сосудистой оболочки глаза. Эти изменения могут приводить к уменьшению внутриглазного объема, а соответственно к увеличению ВГД. В дальнейшем при адаптации организма к условиям, имитирующим космический полет, развивается гипогидратация путем снижения реабсорбции жидкости и электролитов в почечных канальцах, усиления клубочковой фильтрации, и в несколько раз возрастает диурез и выведение осмотических активных веществ, что приводит к нормализации ВГД [8]. Другое возможное объяснение данных изменений заключается в том, что в результате перераспределения жидкости в организме испытуемых происходит повышение выработки внутриглазной жидкости и затруднение ее оттока через дренажную систему глаза [9].

\section{ВЫВОДЫ}

1. При анализе показателей офтальмотонуса во время эксперимента по моделированию гипогравитации были получены статистически достоверные и значимые результаты о повышении уровня ВГД в период с момента начала эксперимента и до 21 суток исследования ( носит транзиторный характер, после окончания воздействия данных условий уровень офтальмотонуса возвращается к исходным показателям. 3. По данным прямой офтальмоскопии, никаких изменений на глазном дне, в частности на ДЗН, у испытуемых во время нахождения в условиях моделирования гипогравитации не происходит. 4. По результатам компьютерной периметрии в ходе эксперимента по созданию измененных условий гравитации значимых изменений зафиксировано не было.

\section{Литература}

1. Rastegar N, Eckart P, Mertz M. Radiation - induced cataract in astronauts and cosmonauts. Graefes Arch Clin Exp Ophthalmol. 2002; (240): 534-47.

2. Thomas H, Mader C, Robert OD, Anastas F. Optic Disc Edema, Globale Flattening, Choroidal Folds, and Hyperopic Shifts Observed in Astronauts after Long - duration Space Flight. American Academy of Ophthalmology Published by Elsevier Inc. 2011; 2058-70.

3. Chylack BE, Peterson LE, Feiveson AH, et al. NASA study of cataract is astronauts (NASA). Report 1: Cross - sectional study of the relationship of exposure to space radiation and risk of lens opacity. Radiat Res. 2009; (172): 10-20.

4. Cucinotta FA, Manuel FK, Jones J, et al. Space radiation and cataracts in astronauts. RADIAT Res. 2001; 460-6.

\section{References}

1. Rastegar N, Eckart P, Mertz M. Radiation - induced cataract in astronauts and cosmonauts. Graefes Arch Clin Exp Ophthalmol. 2002; (240): 534-47.

2. Thomas H, Mader C, Robert OD, Anastas F. Optic Disc Edema, Globale Flattening, Choroidal Folds, and Hyperopic Shifts Observed in Astronauts after Long - duration Space Flight. American Academy of Ophthalmology Published by Elsevier Inc. 2011; 2058-70.

3. Chylack BE, Peterson LE, Feiveson AH, et al. NASA study of cataract is astronauts (NASA). Report 1: Cross - sectional study of the relationship of exposure to space radiation and risk of lens opacity. Radiat Res. 2009; (172): 10-20.

4. Cucinotta FA, Manuel FK, Jones J, et al. Space radiation and cataracts in astronauts. RADIAT Res. 2001; 460-6.

5. Frey MA. Radiation health: mechanism of radiation - induced cataracts in astronauts. Aviat Space Environ Med. 2009; 575-6.

6. Draeger J. Tonometry under microgravity condititions. Norderney Symposium on Scentific Results of the German Spacelab Mission D1. 1986; 503-9.

7. Mekjavic PJ, Eiken O, Mekjavic IB. Visual function after prolonged bed rest. J Gravit Physil. 2002; (9): 31-2.

8. Kergoat H, Lovasik JV. Seven-degree head-down tilt reduces choroidal pulsatile ocular blood flow. Aviation, Space and Enviromental Medicine. 2005; 76 (10): 930-5.

9. Mader TH, Gibson GR, Pass AF, et al. Optic disc edema globe flattening, choroidal folds, and hyperopic shifts observed in astronauts after long-dyration space flight. Ophthalmology. 2011; (118): 2058-69. 